\title{
Technè
}

La science au service de l'histoire de l'art et de la préservation des biens culturels

$44 \mid 2016$

Archives de l'humanité : les restes humains patrimonialisés

\section{Petite rhétorique narrative des restes humains muséaux}

Short narrative rhetoric about human remains in museums

\section{Christelle Patin}

\section{(2) OpenEdition \\ 1 Journals}

Édition électronique

URL : http://journals.openedition.org/techne/925

DOI : $10.4000 /$ techne.925

ISSN : 2534-5168

Éditeur

C2RMF

\section{Édition imprimée}

Date de publication : 1 novembre 2016

Pagination : 14-17

ISBN : 978-2-7118-6339-6

ISSN : 1254-7867

Référence électronique

Christelle Patin, « Petite rhétorique narrative des restes humains muséaux », Technè [En ligne], 44 |

2016, mis en ligne le 19 décembre 2019, consulté le 28 juillet 2020. URL : http://

journals.openedition.org/techne/925; DOI : https://doi.org/10.4000/techne.925

\section{(c)}

La revue Technè. La science au service de l'histoire de l'art et de la préservation des biens culturels est mise à disposition selon les termes de la Licence Creative Commons Attribution - Pas d'Utilisation

Commerciale - Pas de Modification 4.0 International. 
Christelle Patin

\section{Petite rhétorique narrative des restes humains muséaux}

Short narrative rhetoric about human remains in museums

Résumé. Les restes humains des collections muséales sont l'objet supports de narration. Ce court article tente de cerner les grandes lignes de cette construction.

Mots-clés. Agentivité, anthropologie, mémoire, mort, musée, patrimoine, restes humains, science.
Abstract. People's attachment to human remains in museum collections has become a powerful narrative source. This short article attempts to give a broad outline of this narrative structure.

Keywords. Agency, anthropology, memory, death, museum, heritage, human remains, science.
«Il est difficile de contredire les morts. »

Antonio Tabucchi, Tristano meurt, Gallimard, 2004.

Par leur présence pleine mais muette, les restes humains influencent les rapports sociaux et sont des objets-acteurs doués d'agentivité ${ }^{1}$. Perçus comme sujet biographique, ils constituent des supports de narration puissants. Or, si chaque cas semble spécifique, une certaine rhétorique s'est mise en place ; elle donne à voir et à penser ces éléments singuliers et singularisés. Quelles en sont les grandes lignes, voire la grammaire ? Que nous conte-t-elle de l'attachement que les humains leur portent et de sa construction ? La sensibilité accrue des restes humains muséaux tient à leur position-clé, à la croisée des champs du patrimoine, du funéraire ou de la corporéité et de la science. Elle relève d'une synergie de leviers successifs. Ainsi, bien qu'inaliénables, ces éléments corporels sont investis d'enjeux patrimoniaux et deviennent des « lieux de mémoire ». Tandis que dans un même mouvement, la science à l'origine de ces collections déclame sa contribution au patrimoine universel de l'humanité mais porte, dans les discours, la tache indélébile des spoliations coloniales, racismes et nationalismes occidentaux. Ce texte abordera donc tour à tour chacune des grandes lignes de ces constructions narratives et privilégiera la situation française. Notons que l'analyse est à visée de réflexion et n'a aucune ambition d'exhaustivité.

\section{Réécrire le passé scientifique des collections}

Une ligne axiologique tendue entre la vision d'une science au passé raciste à décoloniser et celle d'une science rationaliste universelle marque les discours. Le concept de libre consentement, manié à l'envi, interroge la légitimité du prélèvement du corps, celle de sa transformation en objet scientifique, de sa circulation et de son exposition publique. La victimisation des individus revivifie la dimension émotionnelle de l'espace moral, en toute réduction et anachronisme ; la science, notamment l'anthropologie, y a une place de choix. Or, des mondes séparent l'appréciation de la collecte d'un crâne de Héréro de Namibie, exterminés par les colons venus d'Allemagne ${ }^{2}$ dans un contexte de conquête, de celui d'un malade sans lien familial décédé dans un hôpital parisien ou encore d'un don de corps à la Société d'autopsie mutuelle ${ }^{3}$. Notons que sur les 18000 crânes conservés au Muséum national d'histoire naturelle $(\mathrm{MNHN}), 41 \%$ proviennent de France ou d'Europe ${ }^{4}$. Cela ne nie en rien la nécessité d'une analyse plus poussée de l'intrication de la science, à travers ses institutions, hommes et productions, dans les enjeux et processus coloniaux ou de domination en général. Une meilleure connaissance des conditions de collecte des restes humains montre la diversité et la complexité des situations et des enjeux, le poids de la composition des acteurs (occidentaux et locaux) entre eux et avec le politique, les résistances et conflits ${ }^{5}$. En témoignent les lectures de la collecte de la tête du chef néo-calédonien Ataï présentes dans l'espace public (médiatique et législatif). Elles stigmatisent le médecin de marine Jules Navarre, donateur, et l'anthropologue Paul Broca ${ }^{6}$, donataire, niant sa véritable dimension politique par une mise à prix du gouverneur et un confinement politique à l'hôpital militaire des

Christelle Patin, chercheuse associée au centre Alexandre Koyré, Paris (patin.christelle@orange.fr). 
restes prélevés au combat (tête et main) ; l'acheminement en métropole par le scientifique n'étant finalement que le dernier maillon de ce processus. L'histoire des collections est loin d'être un récit linéaire stéréotypé et mérite d'être davantage explorée et circonstanciée. Le non-respect de la libre destinée funéraire par la collecte scientifique amène les vivants à rétablir ce droit. C'est ainsi qu'en accord avec ses dernières volontés, le géant Byrne (fig. 1), décédé à la fin du XVIII ${ }^{\mathrm{e}}$ siècle, fut immergé en mer en 2011 par le Hunterian Museum du Royal College of Surgeons de Londres ${ }^{7}$. Cependant, les témoignages historiques sont rares ; en leur absence, il devient manifeste qu'un glissement s'effectue du libre consentement de la personne, au nom de sa liberté, à la question de la dignité de l'humain en général. L'évaluation morale n'est pas aisée car ce qui relève de la volonté de l'individu ne l'est pas toujours du point de vue de celle de la famille ou des communautés et vice-versa. Rappelons que le droit de libre destinée de sa dépouille mortelle repose sur la loi du 15 novembre 1887. Par ailleurs, la vente du cadavre d'un proche ou son abandon à la science furent des stratégies économiques ou idéologiques effectives au XIX ${ }^{\mathrm{e}}$ siècle.

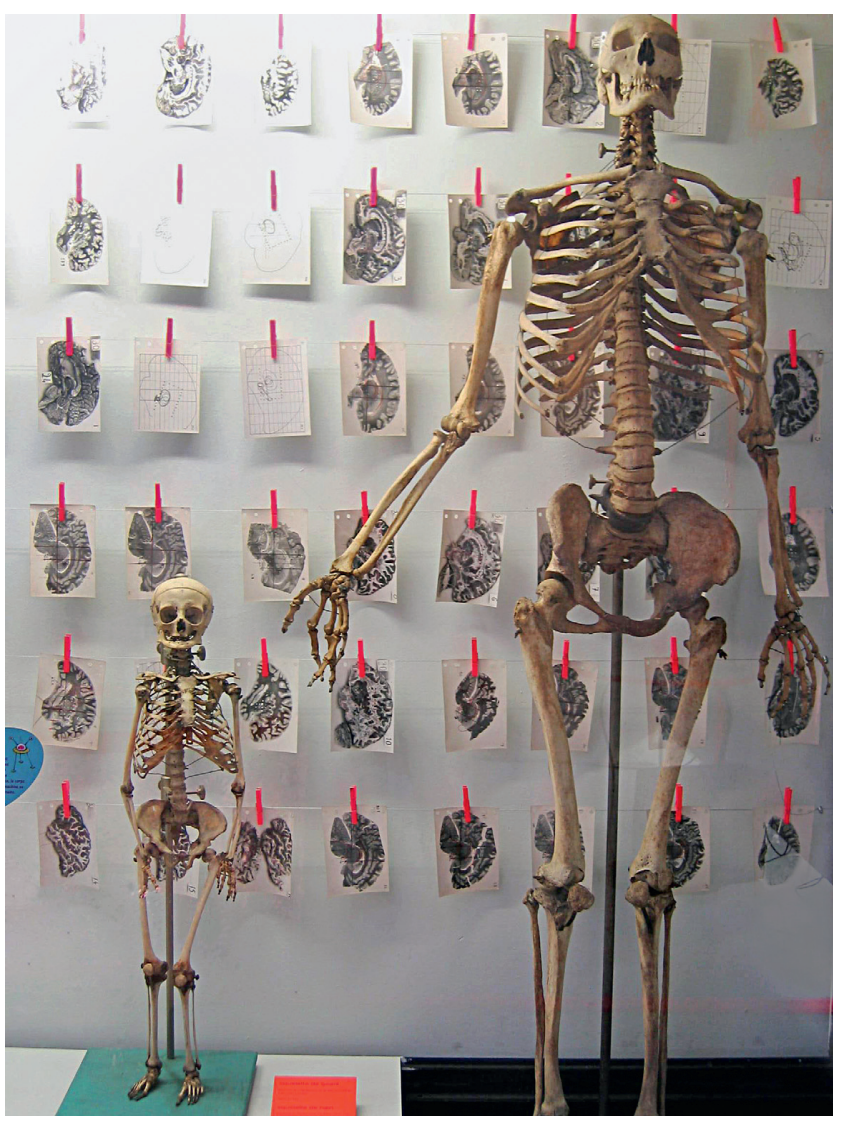

Fig. 1. Le squelette de ce géant fut livré par le gardien du cimetière avec une contrepartie financière de 200 francs, montant qui correspondait à l'achat d'un squelette humain sur le marché des pièces anatomiques en cette fin $\mathrm{du}_{\mathrm{XIX}}{ }^{\mathrm{e}}$ siècle. Dans un courrier, le père de celui-ci réclama également son dû au scientifique du Muséum et non le retour et la réinhumation des restes. (C) Musée de l'Homme/C. Patin.

\section{Réécrire le présent scientifique des collections}

En France, la caricature du scientifique peu responsabilisé aux enjeux de ces pièces spécifiques perdure. Elle est entretenue dans les discours médiatiques et officiels, tantôt par l'accent porté sur une gestion peu rigoureuse des collections, tantôt par la valorisation d'acteurs « secondaires » qui masque les réflexions effectives menées par ces professionnels depuis plus de dix ans. L'épisode de la remise officielle du crâne d'Ataï (fig. 2), le 28 août 2014, et de sa « redécouverte » en 2011, est emblématique. Pour le directeur du MNHN, il n’y avait eu aucune « redécouverte »; le crâne avait toujours été conservé dans les collections de la Société d'Anthropologie de Paris (SAP) hébergées par le Muséum. Or, dans le discours suivant, la ministre des Outre-mer mettait à l'honneur l'écrivain Didier Daeninckx en tant que "découvreur d'Ataï ", réactivant l'idée de perte, déjà instrumentalisée lors de l'épisode de la «Vénus hottentote » en 2002. Cette figure médiatique, incarnant par ses écrits les justes luttes postcoloniales, fut associée à celle de José Bové sollicité par les Kanaks en tant que député européen. Tous deux furent désignés comme les principaux acteurs ayant ouvré à ce retour. À aucun moment, cette mise en scène ne mentionna les scientifiques du Muséum ou ceux de la SAP. Pourtant, un important travail de réflexion avait été mené en amont sur le statut et le devenir des collections, ainsi que sur la faisabilité juridique d'un tel retour $^{8}$. En définitive, ces relectures au symbolisme puissant permettent de se construire une identité contemporaine dans la continuité culturelle des peuples opprimés et leur reconnaissance, groupes spoliés, exclus et hiérarchisés par la science. Le retour à la terre serait la seule " place décente de repos », loin du «purgatoire légal des étagères muséales ${ }^{9}$ ", comme en témoignent les démarches d'inhumation de restes non documentés et non affiliés du National Museum for the American Indian et du Denver Museum of Nature and Science. D'une autre façon, la question de la réinhumation des restes issus des fouilles archéologiques après étude anthropologique se pose aussi en France. Elle interroge tant l'éthique que la constitution des collections de recherches soumises à un mouvement de déflation ${ }^{10}$. Tantôt l'étude scientifique, en amont de la réinhumation, est jugée indigne. Ce fut le cas de l'étude génétique des restes de Saartjie Baartman en 2002, qui fut demandée par les scientifiques de la commission sudafricaine de rapatriement et finalement rejetée ${ }^{11}$. Pourtant, elle aurait pu permettre l'établissement d'une filiation avec des familles de même patronyme ou une meilleure connaissance de son origine. Mais en tant qu'ancêtre fondateur d'une nouvelle Afrique du Sud enfin pacifiée, son appropriation ne pouvait être que collective. Tantôt le processus d'authentification préalable aux restitutions génère de nouvelles investigations scientifiques : approfondissement de la documentation historique, analyse bio-anthropologique et numérisations. Ces nouvelles données nourrissent le récit et, parallèlement, l'attachement porté au reste ; elles lui donnent de la consistance. Cette logique est attestée par le récent rapport de politique de rapatriement du gouvernement du Nunatsiavut 
issu d'une consultation publique des populations inuits ${ }^{12}$. Cependant, le temps politique de la restitution n'est pas toujours soumis à la même dynamique que celui de l'étude scientifique. La compréhension de la biographie culturelle parfois complexe, inhérente à l'établissement d'une documentation solide, nécessite du temps. Le musée Te papa de Wellington en a fait l'expérience et s'interroge en 2013 sur l'authenticité d'une toï moko (tête maorie momifiée et tatouée) restituée par la France, peu documentée, dont les inscriptions « Malais, maorie, Nouvelle Zélande » semblent apocryphes et dont le cartel indique « Malais tahitien de Toetoe».

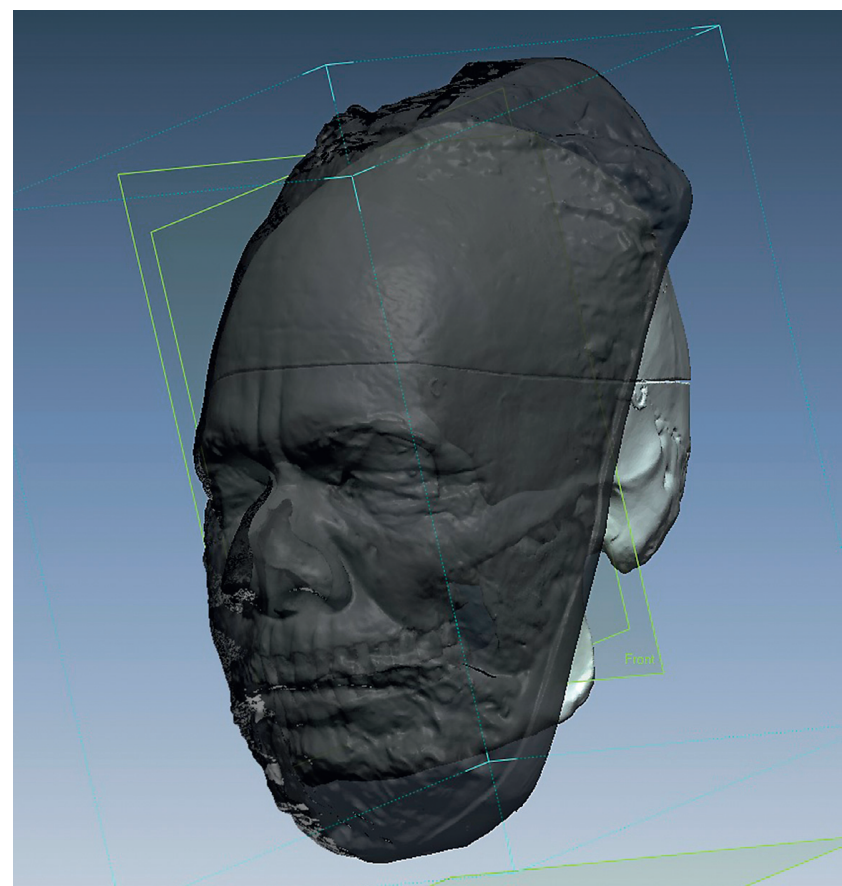

Fig. 2. Superposition tridimensionnelle du masque mortuaire d'Ataï et de son crâne sec. ( ) Plateforme Surfaçus/MNHN.

\section{La construction d'une communauté « morte vivante »}

Comme l'indique le sociologue Arnaud Esquerre, l'évolution récente du rapport au corps mort tend à agglomérer vivants et morts au sein d'une même entité politique, la communauté «morte-vivante", dont l'identité est ancrée dans un territoire ${ }^{13}$. Entre 1970 et 1980, les revendications des mouvements autochtones sur les droits du sol et de liberté religieuse (dont les rites funéraires) se sont concentrées sur le retour ou l'accès au patrimoine culturel, incluant les restes humains. La construction d'une filiation avec ces restes mués en ancêtres par réactivation d'attachements anciens ou bien leur réinvention nourrit la continuité. La terre des ancêtres est assignée à un territoire délimité par l'État. Dans cette perspective, l'identité collective, surdéterminée par une vision culturaliste, préexiste à l'individu. L'inhumation des restes donne du corps au lieu et administre la preuve de son âme. Tandis que, tel un reliquaire, l'espace sacralise les vestiges humains sertis dans l'histoire locale, indissociable d'une temporalité reconstituée, vécue comme quête des origines. L'exemple des restes de «Buhl woman » en Idaho datée de 10800 ans est marquant et s'inscrit dans la politique proactive de rapatriement des « Native American ». Son squelette fut rapatrié dans la tribu Shoshone-Bannock dont la tradition orale ancre l'origine des ancêtres sur le sol américain depuis un temps immémorial ${ }^{14}$. Dans un contexte de droit à l'autodétermination, la reconnaissance des traditions orales s'impose au détriment de l'administration scientifique de la preuve d'une filiation, d'ailleurs peu aisée à établir sur une durée aussi longue. Quels sont les éléments présents dans les discours et pratiques qui permettent de cimenter cette communauté qui lie vivants et morts ? Le principal étai est la commune nature ontologique de ses composants. Comme le signale un intervenant lors d'un colloque sur les collections anatomiques en 2012 : «Je suis celui qui regarde et je suis regardé par le corps que je regarde, une sorte d'humain en fraternité ${ }^{15}$. » Cette approche, toute éthique, devait interpeller le visiteur sur un possible appel au voyeurisme. Cependant, elle inscrit l'élément de corps humain dans une temporalité partagée avec les vivants et construit une sorte d'humanité atemporelle, voire aculturelle, au présentisme permanent. La création et l'imposition de la dénomination « restes humains » en tant que catégorie normative, initialement exogène au monde muséal et scientifique (à l'exception sans doute de l'archéologie), répondent à cette exigence morale ${ }^{16}$. Elle balaye les autres catégories historiques usuelles, qu'elles soient anatomiques, artistiques, ethnologiques ou plus utilitaires. Pourtant, elle regroupe des matérialités bien hétérogènes, que ce soit en termes d'échelle corporelle, de modifications ante ou post-collecte générant des corporéités hybrides, d'utilisation et d'âge. La dimension ontologique de cette approche catégorielle qui dépasse la dimension culturelle de ces éléments prend sens dans un système d'équivalence : la partie, quelle qu'elle soit, vaut pour le tout. Il s'ensuit une sollicitation des valeurs d'équité, d'intégrité et de compassion au service du respect de la dignité humaine. Outre une dimension totale agrégeant vivants et morts, chaque communauté donne une place majeure à la filiation et à la «pureté » culturelle. Si bien qu'une double appartenance ou un mélange entre elles semblent ardus. L'homme communautaire est difficilement celui d'une histoire naturelle.

Finalement, tout comme le récit d'une histoire linéaire et stéréotypée des collections, la dignité précédemment affichée est rapidement mise à l'épreuve dans la pratique. L'agentivité des restes humains s'avère inféodée à l'attachement qu'ils suscitent. C'est ainsi que la peau tannée et cirée du Sud-Africain naturalisé «El Negro », conservé au musée Darder de Banyoles, ne fut pas restituée en 1996, contrairement à son crâne et autres os des membres. Sa détérioration lors du démontage du corps et son état modifié par le travail naturaliste en faisaient, en dépit de son ontologie, une pièce indigne du devoir 
de mémoire et de l'acte de réparation. Dans un autre contexte, en dépit d'une semblable destinée de violence martiale et de domination, le compagnon d'Ataï ne fit, dans un premier temps, l'objet d'aucune demande de restitution, contrairement au leader historique. L'absence d'investissement politique et familial le concernant mettait à mal le discours politico-moral d'équité et de justice déployé pour légitimer le juste retour des restes. Ainsi, en dépit de ce qu'elle affiche, la communauté « morte-vivante » n'est pas exempte de hiérarchisation. La vie politique des corps morts sert à légitimer de nouvelles élites ${ }^{17}$ et reconfigure les groupes stigmatisés au sein d'un état réunifié. L'intégration de l'érosion du souvenir familial, ses possibles reviviscence et instrumentalisation politique se matérialisent aussi par un traitement muséal différentiel entre les restes identifiés et ceux anonymes, notamment au sein de la collection d'anthropologie du MNHN. L'identification donne une sensibilité accrue aux conditions de conservation, de consultation scientifique, d'exposition ou de diffusion publique et aux demandes de restitution. Il s'agit d'une lecture juridique de la dignité muséale ${ }^{18}$, fondée sur la catégorisation sociale des restes humains et leur degré d'agentivité. Par ailleurs, au-delà des éléments corporels, la prise en compte des capacités de supports, tels la photographie ou le moulage, à incarner la personne et à agir amène certains scientifiques à les intégrer dans la catégorie «restes humains ${ }^{19}$ »; cela implique de communes contraintes quant à leur usage. Les discussions autour de l'exposition du moulage de Saartjie Baartman en sont une illustration. Ce bref aperçu de la production d'une rhétorique normative, souvent naturalisée et globalisée, exclut la clé de voûte de l'agentivité, celle de l'importance du contexte qui livre tout le sens de la capacité d'agir des êtres et des choses.

\section{Notes}

1. L'agentivité est issu de l'anglais agency, popularisé notamment par Janet Hoskins lors d'études sur les biographies d'objets, 2006. Ce concept interroge ce que signifie agir socialement entre personnification et chosification des objets. Voir aussi Bonnot, 2014.

\section{2. http://www.rfi.fr/}

afrique $/ 5 \mathrm{~min} / 20111001$-siecle-apres-leurextermination-allemagne-restitue-cranespeuples-herero-nama

3. La Société d'autopsie mutuelle fut créée en 1876 essentiellement par des membres de la SAP. Elle permettait la donation de corps de personnes connues dont on réalisait ensuite l'autopsie, essentiellement cérébrale. La lutte pour la pratique de l'autopsie allait de pair avec le vif débat du choix de l'enterrement civil ou religieux abouti dans la loi du 15 novembre 1887, prémices à la reconnaissance de la crémation (loi de 1889) puis à la loi de 1905 de séparation des églises et de l'État.

4. Froment, Mennecier, 2014, p. 318.

5. Entre autres, Patin, 2013, Rivet, 2014.

6. Les exemples sont nombreux, voir

«Un chef revient parmi les siens », Le Monde, 10 août 2013.

7. https://www.theguardian.com/ science/2011/dec/22/irish-giant-skeletonmuseum-display

8. Marchal, 2016.

9. Pour reprendre les expressions respectives des acteurs des deux musées, Jenkins, 2016, p. 306-307.

10. Note-circulaire du 19 juillet 2012 relative à la problématique des matériels d'étude et à la méthodologie préalable à l'affection de certains de ces biens aux collections des musées de France, ministère de la Culture.

11. Crais, 2011, p. 160-161.

12. "What we heard": a report on consultations relating to repatriation in
Nunatsiavut, 2016, Nunatsiavut Government, Brake J., 25 p. Lors des consultations, $76 \%$ des personnes interrogées se sont dites favorables à une étude avant inhumation, avec accord de la famille, pour certifier ou non la filiation aux Inuits et l'appartenance culturelle. En revanche, à la question d'un possible prélèvement de pièces anatomiques pour conservation, la moitié y est favorable sous certaines conditions, l'autre moitié reste indécise. Cette consultation fait suite à une étude historique réalisée par France Rivet sur le sort d'Abraham et de ses compagnons exhibés et décédés de la variole à Berlin et Paris.

13. Esquerre, 2011, p. 305-311

14. Jenkins, 2016, p. 303-305.

15. Colloque sur « les collections anatomiques : de la connaissance à la mise en valeur ", du 7 au 9 novembre 2012, Montpellier, INP.

16. L'histoire de la construction et circulation de cette catégorie normative reste à faire à ma connaissance. Celle-ci se diffuse après la Seconde Guerre mondiale et met l'accent non plus sur la dimension résiduelle du reste, comme dans la sphère funéraire, mais sur son humanité. Les restes humains sont avant tout humains avant d'être restes. Provient-elle en France de la diffusion anglosaxonne de human remains? Ces questions méritent d'être davantage explorées.

17. Verdery, 1999.

18. Leca, 2012, p. 649-662, l'auteur distingue les restes anonymes purement archéologiques (comme Ötzi), les restes humains « fortement personnalisés mais (...) frappés d'une désaffection familiale et d'une érosion du souvenir » et enfin les « restes socialement sensibles pour des raisons d'ordre culturel, religieux ou politique qui doivent jouir d'une protection maximum » (comme les cendres de Rudolf Hess ou les têtes maories).

19. Froment, Mennecier, 2014, p. 315

\section{Bibliographie}

Bonnot T., 2014, L'attachement aux choses, Paris, CNRS, 239 p.

Crais C., Scully P., 2011, Sara Baartman and the Hottentot Venus. A ghost story and a biography, Princeton University Press, 232 p.

Esquerre A., 2011, Les os, les cendres et l'État, Fayard, 330 p.

Froment A., Mennecier P., 2014, «Les collections de restes humains, archives de l'humanité ", dans Penser, classer, administrer, pour une histoire croiser des collections scientifiques, Daugeron B., Le Goff A., Paris, MNHN, CTHS, p. 315-333.

Hoskins J., 2006, "Agency, biography and objects", Handbook of material culture, Tilley and alii. (eds.) London, Sage publications, p. 74-84.

Jenkins T., 2016, "Burying knowledge. The fate of Human remains", Keeping their marbles, Oxford University Press, p. 290318.

Leca A., 2012, «De miraculus mortuorum: des droits de l'homme après la mort? L'exemple des restes humains dans les collections muséales ", RRJ, p. 649-662.

Marchal F., Nivart A., Fort A., Ardagna Y., Grimaud-Hervé D., 2016, « La restitution des têtes osseuses d'Ataï et de son compagnon ", BMSAP 28, p. 100105 .

Patin C., 2013, « Les vies post-mortem de Saartjie Baartman, muséologie et économies morales ", dans La Vénus hottentote entre Barnum et Muséum, Blanckaert C. (dir.), Paris, MNHN, p. $65-165$.

Rivet F., 2014, Sur les traces d'Abraham Ulrikab, Horizons polaires, Gatineau, 360 p.

Verdery K., 1999, The political lives of dead body, Columbia University press, $185 \mathrm{p}$. 